\title{
Current status of the JET ITER-like Wall Project
}

\author{
G F Matthews ${ }^{1,2}$, P Edwards ${ }^{1,2}$, H Greuner ${ }^{1,3}$, A Loving ${ }^{1,2}$, H Maier ${ }^{1,3}$, \\ Ph Mertens ${ }^{1,4}$, V Philipps ${ }^{1,4}$, V Riccardo ${ }^{1,2}$, M Rubel ${ }^{1,5}$, C Ruset ${ }^{1,6}$, \\ A Scmidt ${ }^{1,4}$, E Villedieu $^{1,7}$ and JET-EFDA contributors to the ITER-like \\ Wall Project $^{1,2,3,4,5,6,7,8,9}$
}

${ }^{1}$ JET-EFDA Culham Science Centre, Abingdon OX14 3DB, UK

${ }^{2}$ Euratom/UKAEA Fusion Association, Culham Science Centre, Abingdon, UK

${ }^{3}$ Max-Planck-Institut für Plasmaphysik, EURATOM Association, 85748 Garching, Germany

${ }^{4}$ Forschungszentrum Jülich, Euratom Association, Jülich, Germany

${ }^{5}$ Alfvén Laboratory, Royal Institute of Technology (KTH), Association EURATOM-VR, 10044

Stockholm, Sweden

${ }^{6}$ Nat. Inst. for Laser, Plasma and Radiation Physics, Association Euratom-MEdC, Bucharest, Romania

${ }^{7}$ Association Euratom-CEA, Cadarache, DSM/DRFC, Saint Paul Les Durance, France

${ }^{8}$ Association Euratom/SCK-CEN, Boeretang 200, 2400 Mol, Belgium

${ }^{9}$ EFDA-Close Support Unit, Culham Science Centre, Abingdon, UK

E-mail: gfm@jet.uk

Received 28 October 2009

Accepted for publication 5 November 2009

Published 30 December 2009

Online at stacks.iop.org/PhysScr/T138/014030

\begin{abstract}
This paper presents an overview of the status and relevant technical issues for the ITER-like Wall Project with emphasis on progress since the 11th International Workshop on Plasma-Facing Materials and Components for Fusion Applications.
\end{abstract}

PACS numbers: 52.40.Hf, 52.55.-s, 28.52.-s, 28.52.Fa

(Some figures in this article are in colour only in the electronic version.)

\section{Introduction}

The JET ITER-like Wall $[1,2]$ will combine bulk beryllium main-chamber limiters and main protection with a full tungsten divertor. This is the materials combination now selected for the deuterium, tritium (DT) phase of ITER. Experiments in JET will provide the first fully representative test of material migration, material mixing and consequent tritium retention under ITER relevant conditions. Equally important is the opportunity to develop fully integrated scenarios and control schemes for protecting the wall. The project will therefore provide essential information for interpreting material behaviour in ITER and a sound technical basis for guiding the development of ITER scenarios.

Preparations for the installation of the ITER-like wall in JET are now well advanced. All manufacturing prototypes for the main-chamber tiles have been assembled and series manufacture of beryllium components has begun. Meanwhile, a second long remote handling boom has been assembled and commissioned which will allow minimization of the shutdown time through supply of components and tools at the point of work. This paper aims to give an overview of the status of the manufacturing of the new first wall components and the preparation of remote handling tooling, procedures and equipment. There are many valuable lessons to be learned both from the design and technical development to the need to integrate remote handling into the design right from the start of the project.

\section{Current status}

At the time of writing, all the manufacturing prototypes have been assembled and accepted (figure 1) and series production of the 86000 parts is in progress in all areas with about $25 \%$ completed overall. The parts are procured in a number of different contracts and tile carriers are loose assembled at JET before being sent to the beryllium machining companies for final assembly and inspection on representative jigs. The shutdown to install the new wall is scheduled to start towards 


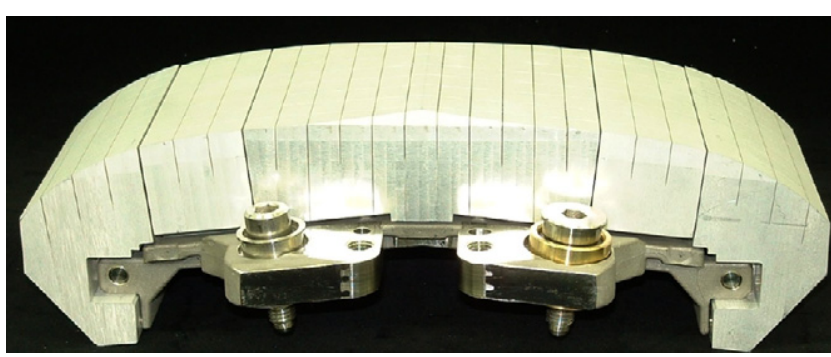

Figure 1. Manufacturing prototype of an inner wall guard limiter tile with the tiles made from aluminium rather than beryllium but using the same processes. The tile assembly is $290 \mathrm{~mm}$ wide.

the end of 2009 with pump-down of the vessel by the end of 2010. Although the logistics for supply of the tiles still looks challenging, the current critical path lies through the remote handling preparations. A lesson for ITER is that it is very easy to underestimate the magnitude of the effort required to ensure that the both the plasma facing component design is installable remotely and that all aspects of the remote handling system are fully prepared. For the ITER-like Wall, the manpower required to design and procure the in-vessel components is very comparable to that required to design and procure the remote handling tools and mock-ups, and to prepare installation procedures. For the scientific exploitation of the new wall, the full set of long-term samples is being replaced and optimized for use with the new wall and this work is advancing well $[8,10]$.

\section{Technical issues}

The basic technical design requirements and solutions were previously described at the 11th International Workshop on Plasma-Facing Materials and Components for Fusion Applications [1,2] and so will not be repeated here. Since this time there have been a number of significant developments that are outlined in the following sub-section.

\subsection{Tungsten coating of divertor and special main-chamber tiles}

Since the original $\mathrm{R} \& \mathrm{D}$ on tungsten coating technologies for the JET divertor [4], considerable further development has been required. A $200 \mu \mathrm{m}$ vacuum plasma spray (VPS) process was originally selected for the divertor on the basis of the performance of the small samples that had to be used at that time. It was known that the anisotropic thermal expansion mismatch between the 2D carbon fibre composite (CFC) material used in JET and the tungsten coating was a significant risk for thick coatings in particular. Changing the divertor material was not an option due to time and the design effort it would have required. So, when the VPS process did not scale up to full size tiles, a $14-30 \mu \mathrm{m}$ thick W/Re physical vapour deposition (PVD) multi-layer coating was developed and tested in the GLADIS ion beam facility [2]. However, although the initial results appeared good, there was some unexpected behaviour [5]. The first most obvious problem was a strong discoloration of tiles coated with a W/Re multilayer after storage in air. This has been attributed to rhenium oxide which appears to migrate to the surface through pores in the coating, an effect not seen with fine

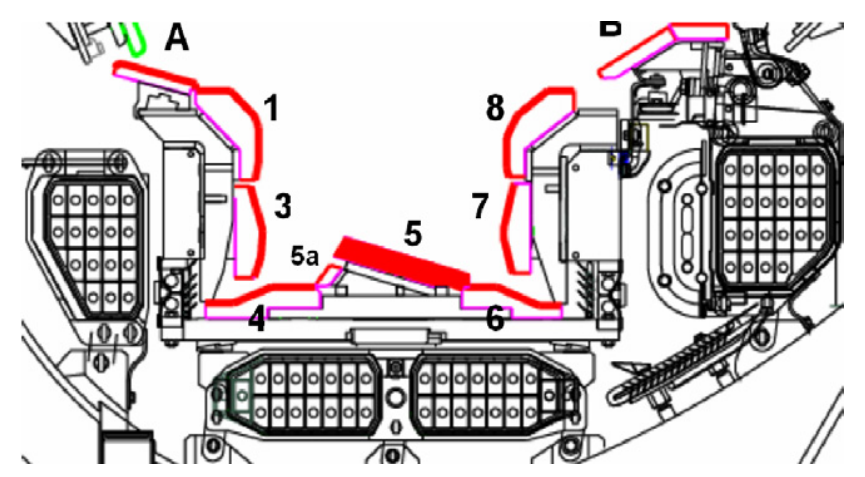

Figure 2. New divertor coating configuration is as follows: Tiles A, 3, 4, 5a, B and C-12 $\mu \mathrm{m} \mathrm{Mo/W}$. Tiles $1,6,7$ and $8-25 \mu \mathrm{m} \mathrm{Mo/W}$ or $\mathrm{Mo} / \mathrm{W} / \mathrm{Mo} / \mathrm{W}$. The Mo layers are $\sim 2 \mu \mathrm{m}$ thick in all cases and all produced by the CMSII process [9]. Tile 5 is a bulk W design [3].

grained graphite (FGG). The second was the discovery that the carbidization at high temperature was more extensive with the multi-layer structure than with a pure tungsten coating - again this result was contrary to the data with FGG substrates. There was a clear evidence therefore that the rhenium posed a risk to the coating performance and hence tests were carried out on vacuum arc-deposited tungsten only coatings. These coatings performed fairly well (but still with a few small local buckles) on small samples coated with $25 \mu \mathrm{m}$ of tungsten. However, when full size production tiles were coated, there were a substantial number of buckles visible after the manufacture which extended along some in-plane fibres. The frequency of these defects depended strongly on the tile geometry and local fibre orientation. Tests were therefore carried out on divertor tiles coated using the combined magnetron sputtering and ion implantation (CMSII) process [9] which was previously selected only for coating main-chamber CFC tiles. These coatings have an Mo inter-layer and $\mathrm{W}$ top coat and have consistently shown the best resistance to local buckling of any process studied. Details about the influence of the Mo on the coating characteristics are given in poster P15 (this conference). Real divertor tiles have been coated and tested in GLADIS both with the standard thickness of $12 \mu \mathrm{m}$ and double thickness coatings. These tests have proven very successful and series production has now started on the divertor tiles requiring a standard thickness coating. More than $50 \%$ of Tile $4 \mathrm{~s}$ (figure 1) are already coated. The best way to achieve a double thickness coating is still being evaluated, the question being whether to repeat the Mo layer or not. The new divertor configuration for this coating is shown in figure 2. Series production of the W-coatings for the main-chamber protection tiles for areas is also progressing well with about $25 \%$ completed.

A conservative estimate for the erosion rate that allows for the effect of surface roughness is that it will be $<0.25 \mathrm{~nm} \mathrm{~s}^{-1}$ [2]. This figure is based on experimental data from tungsten stripes and coated tiles exposed in JET. It is also consistent with the analysis of W-coated tiles from ASDEX-Upgrade and implies a minimum lifetime, due to sputtering, of $100000 \mathrm{~s}$ for the coatings in the outer divertor of JET. Given that the new divertor configuration includes a bulk $\mathrm{W}$ tile [3], this is considered more than sufficient for the JET programme. However, there are other factors that may reduce the lifetime such as fatigue and carbidization [5]. The 


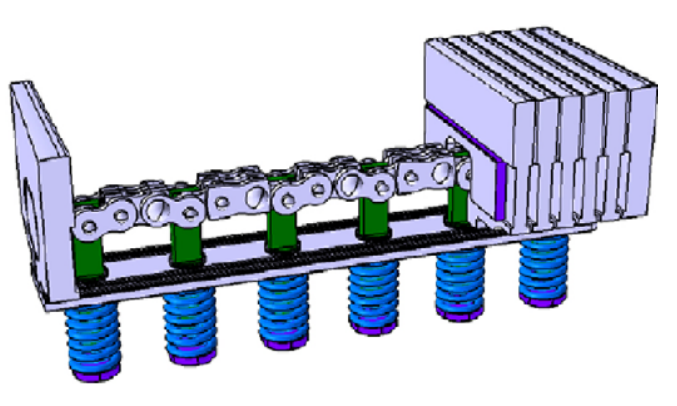

Figure 3. Tensioning system for the bulk W Tile 5 (figure 1) is based on a chain. The resulting stack assembly is $160 \mathrm{~mm}$ long.

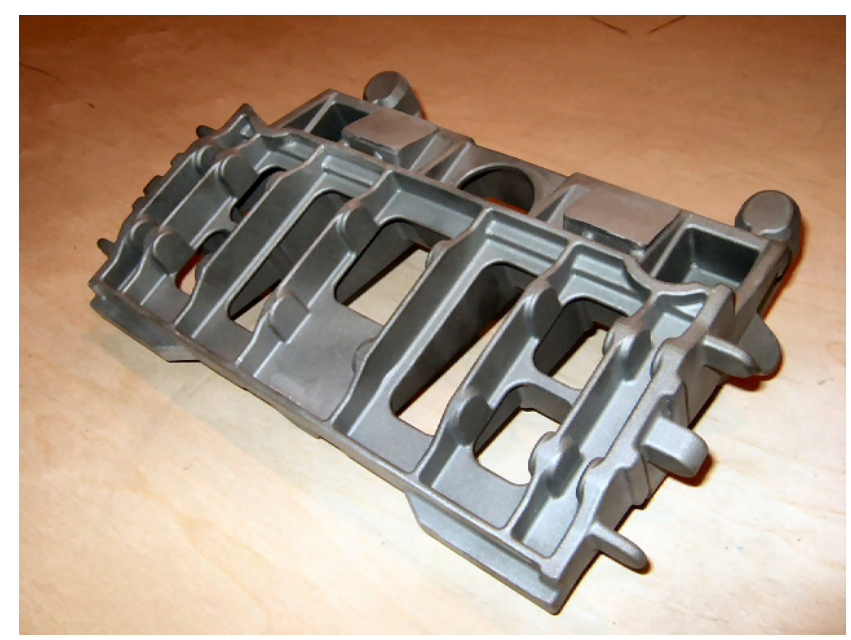

Figure 4. Example of a vacuum cast Inconel 625 outer poloidal limiter carrier before machining (length $293 \mathrm{~mm}$ ).

project now intends to use the JUDITH 2 facility [7] to better characterize these limits experimentally.

\subsection{Bulk W divertor Tile 5}

A low-stress, low eddy force design for a bulk tungsten Tile 5 (figure 2) has been developed based on stacks of tungsten lamellae, $6 \mathrm{~mm}$ thick, compressed together by a Densamet and Nimonic chain, figure 3 [3]. The key technological questions that have been addressed since PFMC11 are the creep of Densamet and relaxation of tensioning springs at high temperatures, and characterization of the mechanical properties of the tungsten grade to be used for the lamellae [3]. Work is ongoing to characterize the energy limits of this design not only experimentally using prototypes in the MARION ion beam facility at FZJ but also using finite element modelling.

\subsection{Vacuum cast Inconel}

Vacuum casting has been used to produce most of the Inconel 625 carriers for the beryllium tile assemblies, figure 4 . This is driven by the economics of machining some geometries from solid. The carrier engineering has to take into account the fact that the cast material is only about half as strong as for wrought (yield strength of vacuum cast $625=200 \mathrm{MPa}$, wrought annealed bar $=410 \mathrm{MPa}$ ). A concern, however, has been that castings always contain voids and it was possible

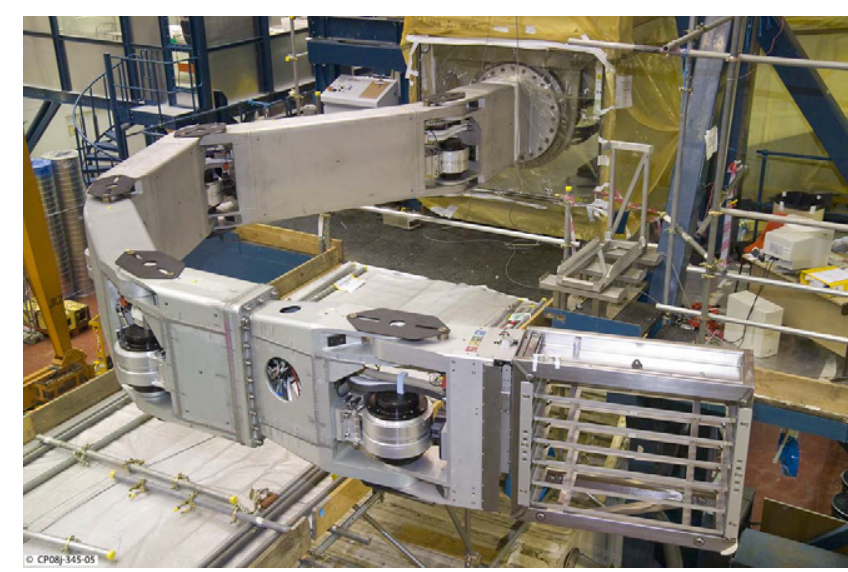

Figure 5. Second JET remote handling boom during commissioning. Its purpose is to deliver tools and components to the area where the main boom and MASCOT manipulator are working [6].

that these would produce virtual leaks. The castings are hot isotatic pressed (HIPed) in an argon atmosphere at a pressure of $100 \mathrm{bar}$ and temperature of $1160^{\circ} \mathrm{C}$. We would expect therefore that such leaks would be characterized by a high argon outgassing level. Vacuum tests are ongoing but so far there is no evidence for any virtual leakage from voids, even from castings that were selected because voids were visible on the $\mathrm{x}$-rays (all castings undergo 100\% x-ray).

\section{Remote handling}

The large numbers of tile assemblies to be replaced in JET $(\sim 5000)$ and the challenging timescale $(\sim 14$ months) has meant that the efficiency of remote handling operations has had to be increased [6]. The existing system was rate limited by the fact that the working boom that supports the MASCOT manipulator had to collect all tools and components from the same port in Octant 1 . To solve this problem, a new boom has been constructed and commissioned (figure 5) whose sole purpose is to transport components and tools to the place of work of the main boom [6]. Mock-up trials are defined as remote handling trials with realistic components in a simulated torus environment. These are used not only validate the remote handling tools and component design but also to develop installation procedures.

Production of the installation procedures is a major task in its own right and is less trivial than it might first appear. The detailed sequence of operations required to fit and use the tooling and assemble the components has to be fully characterized within the operational database so that any boom operator can apply it without experience of handling that particular component. This includes the instructions that directly control pre-programmed moves for the booms and MASCOT. These procedures are validated in a virtual reality (VR) environment which represents the inside of the torus, remote handling equipment and components. The VR model runs in real time in parallel with operation of the real hardware. There were a total of 135 new procedures to be developed for the ILW shutdown, 17 mock-up components, 33 jigs and a total of 280 new pieces of tooling and equipment 
to design and procure. The tooling covers a very wide range in complexity from simple MASCOT fingers to a remotely installed and operated saw.

\section{Conclusions}

The ILW project has made considerable progress since the 11th International Workshop on Plasma-Facing Materials and Components for Fusion Applications with series production of all components underway and major advances in the remote handling preparations. Effort is now also being focused on planning the programme with the new wall and in particular evaluating the new operational limits [11] and developing strategies to work within them. This is particularly important because with the material change and heating upgrade to $35 \mathrm{MW}$ of neutral beams, the wall will become very much easier to damage as will be the case in ITER.

\section{Acknowledgments}

This work was funded jointly by the UK Engineering and Physical Sciences Research Council and by the European Communities under the contract of Association between
EURATOM and UKAEA. It was carried out within the framework of the European Fusion Development Agreement. The views and opinions expressed herein do not necessarily reflect those of the European Commission.

Published under licence from EURATOM.

\section{References}

[1] Matthews G F et al 2007 Phys. Scr. T128 137

[2] Matthews G F et al 2009 Development of divertor tungsten coatings for the JET ITER-like Wall J. Nucl. Mater 390-391 934

[3] Mertens Ph et al 2009 Phys. Scr. T138 014032

[4] Maier H et al 2007 Nucl. Fusion 47 222-7

[5] Maier H et al 2009 Phys. Scr. T138 014031

[6] Haist B, Mills S and Loving A 2008 Remote handling preparations for JET EP2 shutdown Proc. 25th Symp. on Fusion Technology (Rostock, Germany, September 2008)

[7] Schmidt A 2009 Phys. Scr. T138 014034

[8] Widdowson A et al 2009 Phys. Scr. T138 014005

[9] Ruset C et al Proc. 12th Int. Workshop on Plasma-Facing Materials and Components for Fusion Applications

[10] Rubel M et al Proc. 12th Int. Workshop on Plasma-Facing Materials and Components for Fusion Applications

[11] Riccardo V et al 2009 Phys. Scr. T138 014033 